\title{
Hematogenous Osteomyelitis by Acinetobacter Baumannii: Case Report and Literature Review
}

\author{
Rajendrakumar Chimanlal Patel $^{1}$, Sumir Prakash Sahgal ${ }^{1,2}$, Shervin Mortazavi ${ }^{1,2,3}$, \\ Yagnang Kaushikkumar Vyas ${ }^{1}$, Richard Joseph Adam ${ }^{4}$, Vera Salim Antonios ${ }^{1}$
}

${ }^{1}$ Essen Medical Associates, New York, USA; ${ }^{2}$ Department of Internal Medicine, Albert Einstein College of Medicine, New York, USA; ${ }^{3}$ Department of Internal Medicine, Bronx Lebanon Hospital Center, New York, USA; ${ }^{4}$ Department of Radiology, Bronx Lebanon Hospital Center, New York, USA.

Email: doctor17@gmail.com

Received March 24 ${ }^{\text {th }}$, 2011; Revised April 10 ${ }^{\text {th }}$, 2011; Accepted 21 $1^{\text {st }}, 2011$.

\begin{abstract}
Background: Skeletal infection with Acinetobacter baumannii is a rare condition and found mainly among soldiers injured in war. Multidrug resistant (MDR) Acinetobacter baumannii (A. baumannii) osteomyelitis is difficult to treat and requires long course of intravenous antibiotics. Most of reported cases in the literature are the consequences of direct inoculation of the pathogen. Here in, we report the first case of A. Baumannii osteomyelitis disseminated through hematogenous route and the therapeutic approach for this rare infection. Clinical Presentation: A 46 year old African-American male patient with human immunodeficiency virus (HIV) and end-stage renal disease on hemodialysis, who developed persistent MDR A. baumannii bacteremia in the hospital, thought to be secondary to the hemodialysis catheter, necessitating replacement of the catheter. Three months after his discharge to a skilled nursing facility (SNF), he developed left leg swelling without noticeable pain or fever. MRI revealed findings consistent with chronic osteomyelitis of left tibia and intra operative bone culture grew MDR A. baumannii. The patient had good outcome after three surgical debridements and prolonged period (7 Months) of dual antimicrobial therapy. Discussion: While in most documented cases of A. baumannii osteomyelitis, entry appears to require direct inoculation; our case suggests that this pathogen can seed into bone hematogenously in the setting of immunosuppression, persistent bacteremia and possibly in the presence of underlying bone infarcts. Clinicians need to be aware of this rare possible consequence of A. baumannii bacteremia. In conclusion, combination of multiple surgical debridements and dual antimicrobial therapy for a long period may result in a good outcome.
\end{abstract}

Keywords: Osteomyelitis, Hematogenous, Acinetobacter Baumannii, Risk Factors, Bacteremia

\section{Introduction}

A 46 year old African American male patient with HIV/Acquired immunodeficiency syndrome $(\mathrm{CD} 4=6$, Viral load > 60,000), non compliant with antiretroviral medications, hypertension, chronic kidney disease and history of anal squamous cell carcinoma in remission was admitted to a community hospital with fever, reduced oral intake, and diarrhea. During his hospital stay, he was admitted in Intensive Care Unit (ICU) due to respiratory distress requiring intubation and mechanical ventilation. He was diagnosed with acute on chronic renal failure which required urgent dialysis, and right lower lobe pneumonia which was treated empirically with vancomycin, piperacillin-tazobactam and azithromycin. Permacath was placed for future hemodialysis. Blood cultures remained negative. On this antibiotic regimen, he improved, was extubated and later discharged to SNF. Two weeks later, he was transferred back to the hospital with fever and hematuria, treated in ICU with ciprofloxacin for presumed urosepsis, and received blood transfusion secondary to a significant drop in hematocrit. His blood and urine cultures were negative. He was stabilized and discharged back to SNF, only to come back 48 hours later with fever and no obvious source of infection. He was empirically started on vancomycin and piperacillin-tazobactam. Blood cultures collected on admission grew multidrug resistant Acinetobacter baumannii (sensitive to polymyxin and ampicillin-sulbactam) with one set growing vancomycin-resistant Enterococcus faecium as well. He was placed on contact isolation and antibiotics were changed to linezolid, gentamicin and ampicil- 
lin-sulbactam. On day six, repeated blood cultures continued to grow E. faecium and A. baumannii, so treatment was again adjusted to daptomycin, gentamicin and polymyxin. The hemodialysis catheter was suspected to be the source of bacteremia, necessitating its removal, with a placement of a temporary Shiley catheter for hemodialysis. Repeated blood cultures continued to grow A. baumannii intermittently, with positive cultures reported on day 7, day 13, and day 16 and a strain that became intermediately sensitive to ampicillin-sulbactam. Bacteremia finally cleared with the continuation of same antibiotic regimen for four weeks, and a new permacath was placed on day 35. Patient was started on antiretroviral therapy and then discharged back to SNF.

Three months later, he developed left leg swelling without pain or fever. On examination, mild, non-pitting edema and a non-tender bony prominence was noted on proximal part of anterior aspect of left lower leg. Venous duplex ruled out deep venous thrombosis and x-rays showed ill-defined large lytic lesion with periosteal reaction in proximal left tibia (Figure 1). Non contrast computed tomography (CT) scan showed periosteal irregularity, a cortical defect in the antero-medial aspect of the tibia consistent with cloaca, and an expanded medullary cavity with a lobulated $3.3 \times 1.8 \times 11.6 \mathrm{~cm}$ lesion, suggestive of sequestrum (Figure 2). These findings were consistent with chronic osteomyelitis. Magnetic resonance imaging (MRI) without gadolinium showed subcutaneous tissue abscess, prominent surrounding marrow edema and periostitis of proximal left tibia (Figure 1). Osteonecrosis was noted in the left distal femur. Tibial biopsy and culture from initial therapeutic debridement confirmed the diagnosis of osteomyelitis; no acid-fast bacilli or fungal elements found and no evidence of malignancy noted. Cultures grew MDR A. baumannii with similar susceptibility pattern as the strain causing bacteremia during prior hospitalization.

Intravenous polymyxin B and vancomycin were started and continued for 6 weeks. However, purulent discharge from the let anterior leg was noted during the 5th week of antibiotic course. The patient then underwent extensive debridement of left anterior tibia. Intraoperative bone culture grew A. baumannii and methicillin sensitive staphylococcus aureus (MSSA). Hence, vancomycin was discontinued and cefazolin was added while polymyxin B was continued for another 6 weeks. After completion of treatment, purulent drainage stopped and left leg wound was healed. Three weeks later, purulent drainage was again noted with prominence of the bony mass in the proximal left tibia. MRI without gadolinium was performed showing similar findings as in the prior study. Patient underwent 3rd extensive debridement of the left anterior tibia. He was started on polymyxin B

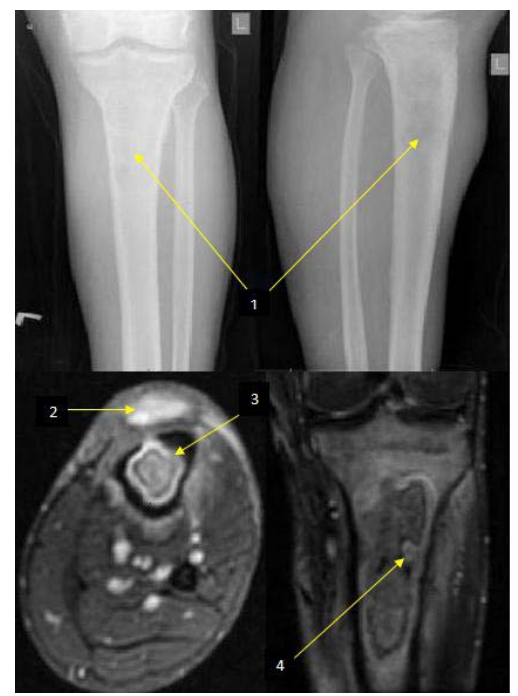

Figure 1. Radiology images of the left leg. A-X-ray AP and Lateral view. 1. Lytic lesion and periosteal reaction.B-Axial T2 weighted MRI, 2. Subcutaneous abscess, 3. Marrow edema and periostealreaction .C MRI (Coronal T1), 4. Sequestrum and Irregularity in periosteum.

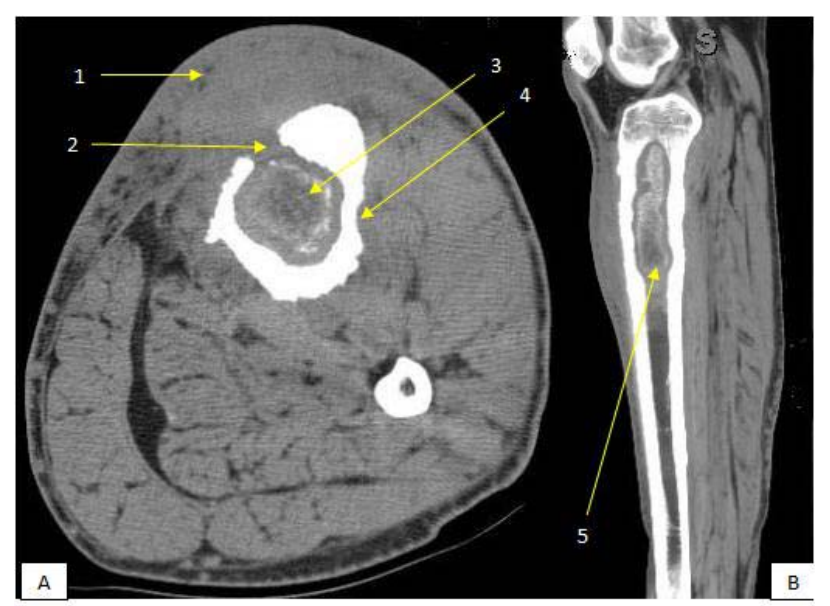

Figure 2. CT scan images of the left leg. A-Axial CT scan, 1. Subcutaneous abscess, 2. Cloaca, 3. Calcified in marrow cavity, 4. Irregular new bone formation (Periosteal reaction); B-Sagittal reformation, 5 . Sequestrum of $8.5 \mathrm{~cm}$.

and ampicillin-sulbactam, which were continued for 3 months, resulting in total healing of the left leg wound.

After one year of follow-up period, patient remains in full recovery in regards to his diagnosis of osteomyelitis.

\section{Discussion}

Over the last two decades, Acinetobacter baumannii has emerged as a major nosocomial pathogen, especially in ICU setting and in immunocompromised patients [1]. The genus Acinetobacter consists of ubiquitous Gram negative, non-fermentative, non-spore forming, aerobic, oxidase negative coccobacilli. A. baumannii has been 
isolated from several sources including environment (soil, water, vegetables, and variety of food products), human skin and throat and respiratory tract of hospitalized patients [2]. Acinetobacter can survive on dry particles up to ten days, and more than four months on some surfaces like PVC (polyvinyl chloride), rubber and ceramics [3,4]. The ability to withstand harsh environmental conditions (including disinfectant solutions and desiccation), and to acquire wide antimicrobial resistance contribute to its propensity for causing outbreaks. In a report by Wilsplinghoff $e$ al. in 49 United States hospitals (24,179 patients) between 1995 and 2002, A. baumannii has accounted for $1.3 \%$ of nosocomial blood stream infection (BSI) and $1.6 \%$ of all nosocomial BSI in ICU settings. Nonetheless, despite its low incidence $(0.6 / 10,000$ admissions), overall mortality, and mortality in ICU setting were $34 \%$ and $43 \%$ respectively [1]. The most common sources of Acinetobacter bacteremia are respiratory tract and intravenous catheters [1]. Risk factors include prolonged hospitalization, ICU stay, mechanical ventilation, recent invasive procedure or surgery, indwelling catheters, broad-spectrum antimicrobial therapy and prior colonization with the organism [2,5-9].

Acinetobacter osteomyelitis has been rarely reported in the literature, mainly in war injury settings. In one report, it was the most frequently found Gram negative isolate from war wounds [10]. Isolated cases of Acinetobacter osteomyelitis were also reported: (1) an 8-year-old boy after hamster bite, treated with parenteral dual antimicrobial therapy (gentamicin and carbenecillin) for 6 weeks [11], (2) a previously injured patient from an armory fragment, causing an open fracture of right femur and treated with parenteral monotherapy (gentamicin) for 6 weeks [12], and (3) a 55-year-old soldier injured by a grenade with osteomyelitis of left proximal femur, treated with parenteral monotherapy (tigecycline) for 43 days [13]. The largest case series reported is by Davis et al. on 23 soldiers wounded in Iraq war who had wound cultures positive for MDR Acinetobacter species [14]. Osteomyelitis was diagnosed in 18 patients who were treated with a combination of antimicrobials and multiple surgical debridements. Dual antimicrobial therapy (amikacin and imipenem) was administered in 10 cases for 6-8 weeks. There was no mortality or recurrence of infection reported within 9 months of follow-up period [14].

A recent pilot study using a rat model suggested that MDR A. baumannii did not appear to cause or contribute to osteomyelitis [15]. However, in our patient, isolation of A. baumannii from several bone specimens, including the ones collected intraoperatively, implies a significant role. Furthermore, the role of MDR A. baumannii in osteomyelitis may be dependent on strains [16]. While the pilot study and the previously reported cases in the lit- erature involved normal hosts, our patient had severe immunosuppression which could be a key factor in the pathogenesis of A. baumannii osteomyelitis. The low virulence of the organism suggested by the study could explain the chronic nature of osteomyelitis in this case. In the experimental rat model, inoculation of S. aureus and A. baumannii was associated with osteolysis, whereas A. baumannii alone lead to osteoblastic activity [15]. The initial bone culture in our patient failed to show $\mathrm{S}$. aureus; however the intraoperative culture grew both $\mathrm{S}$. aureus and A. baumannii. It is unclear if S. aureus bacteremia has preceded the development of A. baumannii infection in this hemodialysis patient. However, it is possible that in the presence of $\mathrm{S}$. aureus, A. baumannii could persist in the bone, leading to a clinically significant osteomyelitis. More studies may be needed to determine the possible synergism between the two organisms.

While in all cases reported in the literature, Acinetobacter osteomyelitis was the result of a direct inoculation, we report the first case of Acinetobacter osteomyelitis resulting from hematogenous spread. Our patient had several risk factors for acquiring nosocomial Acinetobacter infection including immunocompromised status, ICU stay, multiple hospitalizations requiring antimicrobial therapy, and presence of a hemodialysis catheter. After sustaining persistent bacteremia, the catheter was removed and antimicrobial therapy was given for 4 weeks. However, this treatment did not seem to prevent the hematogenous seeding of bacteria in the tibia.

Hematogenous osteomyelitis is primarily a disease of children but can also occur among adults [17]. Involvement of long bones is typically seen in children, while vertebral seeding is common in adults [17]. The risk factors predispose to persistent bacteremia, such as our patient presented with, also favor the development of hematogenous osteomyelitis. It is possible in this case that an underlying condition such as osteonecrosis could have predated the infection and rendered the bone more susceptible to metastatic infection. The large area of sequestration supports this hypothesis. Furthermore, osteonecrosis was noted in the left distal femur on MRI. Potential risk factors in our patient for acquiring osteonecrosis include HIV and renal disease. The radiological appearance in our case is otherwise classical for chronic osteomyelitis, with a cloaca and bony sequestrum.

It is not surprising that multiple surgical debridements were required, with the presence of sequestrum in the setting of chronic osteomyelitis. The typical 6 weeks course of antimicrobials was not sufficient to control the infection. However, failure to eliminate infection could have been associated with the need for further surgical debridements. Polymyxin B has become an important 
antimicrobial agent in the treatment of MDR Acinetobacter species. Nephrotoxicity associated with polymyxin B was not a concern in our case since the patient was already receiving hemodialysis. None of the cases of Acinetobacter osteomyelitis reported in the literature was treated with polymyxin B. The addition of ampicillinsulbactam was beneficial in this case, and dual antimicrobial therapy may have contributed to good outcome. It would be difficult to determine if one factor has made the difference (surgical interventions, duration of antimicrobials, dual therapy) or a combination of all. Nonetheless, our patient responded well with this multifaceted treatment approach.

\section{Conclusions}

In conclusion, this is the first reported case of MDR A. baumannii hematogenous osteomyelitis. While in most documented cases of A. baumannii osteomyelitis, entry appears to require direct inoculation; our case suggests that this pathogen can seed into bone hematogenously in the setting of immunosuppression, persistent bacteremia and possibly in the presence of underlying bone infarcts.

The ability of Acinetobacter to cause metastatic bone infections has not been previously reported and further studies may be warranted. Clinicians need to be aware of this rare possible consequence of A. baumannii bacteremia. In addition, increasing prevalence of this organism worldwide may warrant the need for newer antimicrobials with activity against these organisms. Combination of multiple surgical debridements and dual antimicrobial therapy for a long period may result in a good outcome.

\section{Acknowledgements}

First of all, we like to thank our patient for giving us permission and written consent to collect all the relevant information. We are very thankful to Dr. Alfonso Ortiz for his help throughout the project and Dr. Catherine Maldjian to guide us in selecting appropriate radiological images for this report. We could not have completed this case report without the support from all the administrative staff of medical record and radiological departments of Bronx Lebanon Hospital Center and Montefiore Medical Center.

\section{REFERENCES}

[1] H. Wisplinghoff, T. Bischoff, M. T. Sandra, et al., "Nosocomial Bloodstream Infections in US Hospitals: Analysis of 24,179 Cases from Prospective Nationwide Surveillance Study," Clinical Infectious Diseases, Vol. 39, No. 3, 2004, pp. 309-317. doi:10.1086/421946

[2] P. E. Fournier, "The Epidemiology and Control of Acinetobacter Baumannii in Health Care Facilities,” Clinical Infectious Diseases, Vol. 42, No. 5, 2006, pp. 692-699. doi:10.1086/500202

[3] C. Webster, K. Towner and H. Humphreys, "Survival of Acinetobacter on Three Clinically Related Inanimate Surfaces," Infect Control Hosp Epidemiol, Vol. 21, No. 4, 2000, p. 246. doi:10.1086/503214

[4] C. Wendt, B. Dietz, E. Dietz, et al., "Survival of Acinetobacter Baumannii on Dry Surfaces," Journal of Clinical Microbiology, Vol. 35, No. 6, 1997, pp. 1394-1397.

[5] B. Kurcik-Trajkovska, "Acinetobacter Spp.-A Serious Enemy Threatening Hospitals Worldwide,” Macedonian Journal of Medical Sciences, Vol. 2, No. 2, 2009.

[6] J. M. Cisneros and J. Rodriguez-Bano, "Nosocomial Bacteremia due to Acinetobacter Baumannii: Epidemiology, Clinical Features, and Treatment," Clinical Microbiology and Infection, Vol. 8, No. 7, 2002, pp. 687-693. doi:10.1046/j.1469-0691.2002.00487.x

[7] E. Playford, J. Craig and J. Iredell, "CarbapenemResistant Acinetobacter Baumannii in Intensive Care Unit Patients: Risk Factors for Acquisition, Infection and Their Consequences," The Journal of Hospital Infection, Vol. 65, No. 3, 2007, pp. 204-211. doi:10.1086/503214

[8] P. A. Tiley and F. J. Roberts, "Bacteremia with Acinetobacter Species: Risk Factors and Prognosis in Different Clinical Settings," Clinical Infectious Diseases, Vol. 18, No. 6, 1994, pp. 896-900. doi:10.1093/clinids/18.6.896

[9] J. L. Garcia-Garmendia, C. Ortiz-Leyba, J. GarnachoMontero, et al., "Risk Factors for Acinetobacter Baumannii Nosocomial Bacteremia in Critically Ill Patients: A Cohort Study," Clinical Infectious Diseases, Vol. 33, No. 7, 2001, pp. 933-939. doi:10.1086/322584

[10] M. J. Tong, "Septic Complications of War Wounds,” The Journal of the American Medical Association, Vol. 305, No. 24, 1972, pp. 1044-1047. doi:10.1001/jama.219.8.1044

[11] R. Martin, D. Martin and C. Levy, "Acinetobacter Osteomyelitis from a Hamster Bite,” The Pediatric Infectious Disease Journal, Vol. 7, No. 5, 1988, pp. 364-365. doi:10.1097/00006454-198805000-00020

[12] G. Volpin, N. Krivoy and H. Stein, “Acinetobacter Sp. Osteomyelitis of the Femur: A Late Sequel of Unrecognized Foreign Body Implantation,” Injury, Vol. 24, No. 5, 1993, pp. 345-346.

doi:10.1016/0020-1383(93)90063-C

[13] J. Schafer and J. Mangino, "Multidrug Resistant Acinetobacter Osteomyelitis from Iraq," Emerging Infectious Diseases, Vol. 14, No. 3, 2005, pp. 512-514. doi:10.3201/eid1403.070128

[14] K. Davis, K. Moran, C. McAllister and P. Gray, "Multidrug-Resistant Acinetobacter Infections in Soldiers," Emerging Infectious Diseases, Vol. 11, No. 8, 2005, pp. 1218-1224.

[15] S. C. Collinet-Adler, C. A. Castro, C. G. Ledonio, et al., "Acinetobacter Baumannii Is Not Associated with Osteomyelitis in a Rat Model,” Clinical Orthopaedics and Related Research, Vol. 469, No. 1, 2011, pp. 274-282. doi:10.1007/s11999-010-1488-0 
[16] D. P. Crane, K. Gromov, D. Li, et al., "Efficacy of Collistin-Impregnated Beads to Prevent Multidrug-Resistant A. Baumannii Implant-Associated Osteomyelitis,” Journal of Orthopaedic Research, Vol. 27, No. 8, 2009, pp. 1008-1005. doi:10.1002/jor.20847
[17] J. T. Mader, M. Shirtliff and J. H. Calhoun, “The Host and the Skeletal Infection: Classification and Pathogenesis of Acute Bacterial Bone and Joint Sepsis,” Best Practice \& Research Clinical Rheumatology, Vol. 13, No. 1, 1999, pp. 1-20. doi:10.1053/berh.1999.0003 